\title{
Third Hankel Determinant for A Class of Functions with Respect to Symmetric Points Associated With Exponential Function
}

\author{
GANESH K \\ JMTK, Karimnagar \\ Telangana, INDIA
}

\author{
BHARAVI SHARMA R \\ Kakatiya University \\ Telangana, INDIA
}

\author{
RAJYA LAXMI K \\ TSWRDC, Warangal East \\ Telangana, INDIA
}

\begin{abstract}
The purpose of the present work is to determine the possible upper bound of third order Hankel determinantforthefunctionsstarlikeandconvexwithrespecttosymmetricpointsassociated withexponentialfunctions.

Key-Words: Analytic function, Univalent function, Subordination, Fekete-Szegö inequality, Hankel determinant, Symmetricpoints.
\end{abstract}

Received: October 23, 2019. Revised: April 4, 2020. Accepted: April 17, 2020. Published: April 29, 2020.

\section{Introduction}

The class of all analytic functions $f$ with $f(0)=0$ and $f^{\prime}(0)=1$ in the unit disc $E=\{z \in \mathbb{C}:|z|<1\}$ is denoted by $\mathcal{A}$ and has the Taylor's series expansion of the form given by

$$
f(z)=z+a_{2} z^{2}+a_{3} z^{3}+\cdots
$$

The class of functions that are univalent and analytic in $E$ is denoted by $\mathbb{S}$. The family of functions that are analytic in $E$ and maps $E$ onto the right half plane is denoted by $P$ and functions of this class are of the form

$$
p(z)=1+p_{1} z+p_{2} z^{2}+p_{3} z^{3}+\cdots
$$

For two analytic functions $g$ and $h$ in $E, g$ is subordinate to $h$, denoted as $g \prec h$, if there is an analytic function $w$ in $E$ with $w(0)=0$ and $|w(z)|<1$ such that $g(z)=h(w(z))$, for all $z \in E$. If $h$ is univalent in $E$, the subordination is same as $g(0)=h(0)$ and $g(E) \subseteq h(E)$. Pommerenke ([35], [36]) defined the Hankel determinant $H_{k}(n)$, for positive integers $k, n$ for the functions in $\mathbb{S}$ of the form (1), as below:

$H_{k}(n)=\left|\begin{array}{cccc}a_{n} & a_{n+1} & a_{n+2} \cdots \cdots & a_{n+k-1} \\ a_{n+1} & a_{n+2} & a_{n+3} \cdots \cdots & a_{n+k} \\ a_{n+2} & a_{n+3} & a_{n+4} \cdots \cdots & a_{n+k+1} \\ -- & -- & ------ & --- \\ -- & -- & ------ & --- \\ a_{n+k-1} & a_{n+k} & a_{n+k+1} \cdots \cdots & a_{n+2 k-2}\end{array}\right|$

For fixed positive integers $k$ and $n$ the growth of $H_{k}(n)$ as $n \longrightarrow \infty$ has been determined by Noor [30], with bounded boundary. The Hankel determinant for exponential polynomials was studied by
Ehrenborg [9].The Hankel determinant of different orders is obtained for different values of $k, n$. For instance, when $k=2$ and $n=1$, the determinant

$$
\begin{aligned}
H_{2}(1) & =\left|\begin{array}{ll}
a_{1} & a_{2} \\
a_{2} & a_{3}
\end{array}\right| \\
& =\left|a_{3}-a_{2}^{2}\right|,\left(a_{1}=1\right)
\end{aligned}
$$

This determinant is the particular case of estimating the greatest value of the functional $\left|a_{3}-\mu a_{2}^{2}\right|$ for functions in $\mathbb{S}$, where $\mu$ is real or complex. This is known as the Fekete-Szegö [10] problem. Many researchers like Ali et al. ([1],[2]), Cho and Owa ([6],[7]),Koegh and Merkes [16], Lewandowski et al.[21], Ma and Minda [24], Magesh et al. [25], Murugusundaramurthy et al. [28], Ram Reddy and Sharma [39], Ravichandran et al. [40],Shanmugam et al. [41], Ram Reddy et al.[42], Haripriya and Sharma [12], Srivastava H.M. et al.[43], Tuneski and Darus [44] have studied Fekete- Szegö inequality for various subclasses of univalent analytic functions.

Now for $k=2, n=2$, it can be obtained that

$$
\begin{aligned}
H_{2}(2) & =\left|\begin{array}{ll}
a_{2} & a_{3} \\
a_{3} & a_{4}
\end{array}\right| \\
& =\left|a_{2} a_{4}-a_{3}^{2}\right|
\end{aligned}
$$

The maximum value of $H_{2}(2)$ has been investigated by several authors. For instance the reader can see the work initiated by Hayman [13], Noonan and Thomas [29], Janteng et al. ([14],[15]), Bansal[5], Lee et al. [20], Liu et al. [23], Raina et al. [38], Ohran et al. [31], Laxmi and Sharma [18], Răducanu and Zaprawa [37].Very recently, Zaprawa[47] shown a new direction in estimating the upper bound of the Hankel determinant for $k=2, n=3$, i.e., $H_{2}(3)$ for various 
subclasses of $\mathbb{S}$. This determinant is given by

$$
\begin{aligned}
H_{2}(3) & =\left|\begin{array}{ll}
a_{3} & a_{4} \\
a_{4} & a_{5}
\end{array}\right| \\
& =\left|a_{3} a_{5}-a_{4}^{2}\right|
\end{aligned}
$$

For $k=3, n=1$ the Hankel determinant $H_{3}(1)$ is called as third order Hankel determinant and is given by

$$
\begin{aligned}
H_{3}(1) & =\left|\begin{array}{lll}
a_{1} & a_{2} & a_{3} \\
a_{2} & a_{3} & a_{4} \\
a_{3} & a_{4} & a_{5}
\end{array}\right| \\
& =a_{3}\left(a_{2} a_{4}-a_{3}^{2}\right)-a_{4}\left(a_{4}-a_{2} a_{3}\right)+a_{5}\left(a_{3}-a_{2}^{2}\right)
\end{aligned}
$$

Babalola [4] was the first person to study the upper bound of $H_{3}(1)$ for subclasses of $\mathbb{S}$. Interested readers can see the work carried by several researchers like Vamshee Krishna et al. ([45],[46]), Prajapat et al. ([32],[33]),Altinkaya and Yalcin [3], Cho et al. [8], lecko et al. [19], Kowalczyk et al.[17],Mohd Narzan et al.[27]. Mendiratta et al. [26] introduced and studied the class of starlike functions $\mathbb{S}_{e}^{*}=\mathbb{S}^{*}\left(e^{z}\right)$ defined by

$$
\frac{z f^{\prime}(z)}{f(z)} \prec e^{z},(z \in E)
$$

Very recently, in 2018, Hai-Yan Zhang et al. [11] investigated the upper bound of the Third Hankel determinant for the function class $\mathbb{S}_{e}^{*}$ related with exponential function. The work of above researchers motivated us to determine the non-sharp upper bounds of third Hankel determinant for the class of starlike and convex functions with respect to symmetric points subordinate to exponential function. Now we define the following subclasses.

Definition 1 A function $f \in \mathbb{S}$, is in the class $\mathbb{S}_{s}^{*}\left(e^{z}\right)$ if and only if

$$
\frac{2\left[z f^{\prime}(z)\right]}{f(z)-f(-z)} \prec e^{z}, \text { for all } z \in E .
$$

Definition 2 A function $f \in \mathbb{S}$, is in the class $C_{s}\left(e^{z}\right)$ if and only if

$$
\frac{2\left[z f^{\prime}(z)\right]^{\prime}}{(f(z)-f(-z))^{\prime}} \prec e^{z}, \text { for all } z \in E .
$$

\subsection{Preliminaries}

The lemmas listed below are needed to prove the desired results.
Lemma 3 [34] If $p \in P$, then $\left|p_{n}\right| \leq 2, \forall n \in \mathbb{N}$.

Lemma 4 [22] If $p(z)=1+p_{1} z+p_{2} z^{2}+p_{3} z^{3}+\cdots$, is such that $\operatorname{Re}(p(z))>0$ in $E$, then for some $x, z$ with $|x| \leq 1,|z| \leq 1$, we have

$$
\begin{aligned}
2 p_{2} & =p_{1}^{2}+x\left(4-p_{1}^{2}\right), \text { for some } x,|x| \leq 1 \\
4 p_{3} & =p_{1}^{3}+2 p_{1}\left(4-p_{1}^{2}\right) x-p_{1}\left(4-p_{1}^{2}\right) x^{2} \\
& +2\left(4-p_{1}^{2}\right)\left(1-|x|^{2}\right) z
\end{aligned}
$$

Lemma 5 [40] If $p \in P$, then

$\left|p_{2}-\nu p_{1}^{2}\right| \leq \max \mid\{1,|2 \nu-1|\}$ for any $\nu \in \mathbb{C}$.

\section{Mains Results}

Theorem 6 If $f \in \mathbb{S}_{s}^{*}\left(e^{z}\right)$ then $\left|a_{2}\right| \leq \frac{1}{2}$,

$\left|a_{3}\right| \leq \frac{1}{2},\left|a_{4}\right| \leq \frac{19}{48},\left|a_{5}\right| \leq \frac{13}{24}$.

Proof: As $f \in \mathbb{S}_{s}^{*}\left(e^{z}\right)$ from (9) and using the principle of subordination, we have

$$
\frac{2\left[z f^{\prime}(z)\right]}{f(z)-f(-z)}=e^{w(z)},
$$

Let us define the function $p(z)=\frac{1+w(z)}{1-w(z)}=1+p_{1} z+$ $p_{2} z^{2}+\cdots$, analytic in $E$ with $p(0)=1$ and maps $E$ onto the right half of the $w$-plane. Computing $w(z)$ in terms of $p(z)$, we get,

$$
\begin{gathered}
\begin{array}{c}
w(z)=\frac{p_{1} z}{2}+\left(\frac{p_{2}}{2}-\frac{p_{1}^{2}}{4}\right) z^{2}+\left(\frac{p_{3}}{2}-\frac{p_{1} p_{2}}{2}+\frac{p_{1}^{3}}{8}\right) z^{3} \\
+\left(\frac{p_{4}}{2}-\frac{p_{1} p_{4}}{2}-\frac{p_{2}^{2}}{4}+\frac{3 p_{1}^{2} p_{2}}{8}-\frac{p_{1}^{4}}{16}\right) z^{4}+\cdots
\end{array} \\
\text { Again } e^{w(z)}=1+w(z)+\frac{(w(z))^{2}}{2 !}+\frac{(w(z))^{3}}{3 !} \\
+\frac{(w(z))^{4}}{4 !}+\cdots
\end{gathered}
$$

Substituting (14) in (15), we get

$$
\begin{aligned}
e^{w(z)}= & +\frac{p_{1} z}{2}+\left(\frac{p_{2}}{2}-\frac{p_{1}^{2}}{8}\right) z^{2}+\left(\frac{p_{3}}{2}-\frac{p_{1} p_{2}}{4}+\frac{p_{1}^{3}}{48}\right) z^{3} \\
& +\left(\frac{p_{4}}{2}-\frac{p_{1} p_{3}}{4}-\frac{p_{2}^{2}}{8}+\frac{p_{1}^{2} p_{2}}{16}+\frac{p_{1}^{4}}{384}\right) z^{4}+\cdots
\end{aligned}
$$

Substituting $f(z), f^{\prime}(z)$ on L.H.S. of (13) and replacing $e^{w(z)}$ on R.H.S, upon equating like powers of $z$, we have

$$
\begin{aligned}
& a_{2}=\frac{p_{1}}{4}, a_{3}=\frac{p_{2}}{4}-\frac{p_{1}^{2}}{16}, a_{4}=\frac{p_{4}}{8}-\frac{p_{1} p_{2}}{32}-\frac{p_{1}^{3}}{384}, \\
& a_{5}=\frac{p_{4}}{8}-\frac{p_{1} p_{3}}{16}+\frac{p_{1}^{4}}{384} .
\end{aligned}
$$


Taking modulus on either side of each expression in (17) and applying Lemma 3 and Lemma 5, we obtain

$$
\left|a_{2}\right| \leq \frac{1}{2},\left|a_{3}\right| \leq \frac{1}{2},\left|a_{4}\right| \leq \frac{19}{48},\left|a_{5}\right| \leq \frac{13}{24} .
$$

Theorem 7 If $f \in \mathbb{S}_{s}^{*}\left(e^{z}\right)$ then $\left|a_{3}-a_{2}^{2}\right| \leq \frac{1}{2}$.

Proof: Proceeding as in Theorem 6 and using (17), we have

$$
a_{2}=\frac{p_{1}}{4}, a_{3}=\frac{p_{2}}{4}-\frac{p_{1}^{2}}{16} .
$$

Consider

$$
\left|a_{3}-a_{2}^{2}\right|=\left|\frac{p_{2}}{4}-\frac{p_{1}^{2}}{16}-\frac{p_{1}^{2}}{16}\right|=\left|\frac{p_{2}}{4}-\frac{p_{1}^{2}}{8}\right|
$$

Using Lemma 5, we obtain

$$
\left|a_{3}-a_{2}^{2}\right| \leq \frac{1}{2}
$$

\section{Theorem 8 If $f \in \mathbb{S}_{s}^{*}\left(e^{z}\right)$}

then $\left|a_{2} a_{3}-a_{4}\right| \leq \frac{765+59 \sqrt{118}}{3468}$.

Proof: From equation (17) of Theorem 6, we have

$$
\left|a_{2} a_{3}-a_{4}\right|=\left|\frac{3 p_{1} p_{2}}{32}-\frac{p_{3}}{8}-\frac{5 p_{1}^{3}}{384}\right|
$$

Now applying Lemma 4, we have

$$
\begin{aligned}
\left|a_{2} a_{3}-a_{4}\right|= & \mid \frac{\left(4-p_{1}^{2}\right) p_{1} x^{2}}{32}-\frac{\left(4-p_{1}^{2}\right) p_{1} x}{64}- \\
& \frac{\left(4-p_{1}^{2}\right)\left(1-|x|^{2}\right) z}{16}+\frac{p_{1}^{3}}{384} \mid
\end{aligned}
$$

Denote $|x|=t \in[0,1], p_{1}=c \in[0,2]$. Then, using triangle inequality, equation (23) gives

$$
\begin{aligned}
\left|a_{2} a_{3}-a_{4}\right| & \leq \frac{\left(4-c^{2}\right) c t^{2}}{32}+\frac{\left(4-c^{2}\right) c t}{64} \\
& +\frac{\left(4-c^{2}\right)}{16}+\frac{c^{3}}{384}
\end{aligned}
$$

Suppose that

$$
\begin{aligned}
F(c, 1) \equiv & \frac{\left(4-c^{2}\right) c t^{2}}{32}+\frac{\left(4-c^{2}\right) c t}{64} \\
& +\frac{\left(4-c^{2}\right)}{16}+\frac{c^{3}}{384}
\end{aligned}
$$

Thus we get $\frac{\partial F}{\partial t}=\frac{\left(4-c^{2}\right) c}{64}+\frac{\left(4-c^{2}\right) c t}{16} \geq 0$, the function $F(c, t)$ is non-decreasing for any $t$ in $[0,1]$. This shows that $F(c, t)$ has maximum value at $t=1$.

$$
\begin{aligned}
\operatorname{Max} F(c, t)= & F(c, 1)=\frac{\left(4-c^{2}\right) c}{32}+\frac{\left(4-c^{2}\right) c}{64} \\
& +\frac{\left(4-c^{2}\right)}{16}+\frac{c^{3}}{384}
\end{aligned}
$$

Let us define $M(c):=\frac{\left(4-c^{2}\right) c}{32}+\frac{\left(4-c^{2}\right) c}{64}+\frac{\left(4-c^{2}\right)}{16}+\frac{c^{3}}{384}$, then $M^{\prime}(c):=\frac{3\left(4-c^{2}\right) c}{64}-\frac{11 c^{2}}{128}-\frac{c}{8}$.

$M^{\prime}(c)$ vanishes at $c=r^{*}=\frac{-8+2 \sqrt{118}}{17}$. A simple computation yields that $M^{\prime \prime}(c)<0$, which means that the function $M(c)$ can take maximum value at $r^{*}=$ $\frac{-8+2 \sqrt{118}}{17}$. Hence, we have

$$
\left|a_{2} a_{3}-a_{4}\right| \leq M\left(r^{*}\right)=\frac{765+59 \sqrt{118}}{3468} .
$$

Thus the theorem is proved.

Theorem 9 If $f \in \mathbb{S}_{s}^{*}\left(e^{z}\right)$ then $\left|a_{2} a_{4}-a_{3}^{2}\right| \leq \frac{3}{8}$.

Proof: From equation (17) of Theorem 6, we have

$$
\begin{aligned}
& \left|a_{2} a_{4}-a_{3}^{2}\right| \\
& =\left|\frac{p_{2}}{4}\left(\frac{p_{3}}{8}-\frac{p_{1} p_{2}}{32}-\frac{p_{1}^{3}}{384}\right)-\left(\frac{p_{2}}{4}-\frac{p_{1}^{2}}{16}\right)^{2}\right| \\
& =\left|\frac{p_{1} p_{3}}{32}-\frac{p_{1}^{2} p_{2}}{128}-\frac{p_{1}^{4}}{1536}-\frac{p_{2}^{2}}{16}+\frac{2 p_{1}^{2} p_{2}}{64}-\frac{p_{1}^{4}}{256}\right|
\end{aligned}
$$

According to Lemma 4, we obtain

$$
\begin{aligned}
\left|a_{2} a_{4}-a_{3}^{2}\right|= & \mid \frac{\left(4-p_{1}^{2}\right) p_{1}\left(1-|x|^{2}\right) z}{64}-\frac{\left(4-p_{1}^{2}\right) p_{1}^{2} x^{2}}{128} \\
& -\frac{\left(4-p_{1}^{2}\right) x}{256}-\frac{\left(4-p_{1}^{2}\right)^{2} x^{2}}{64}-\frac{p_{1}^{4}}{1536} \mid .
\end{aligned}
$$

Denote $|x|=t \in[0,1], p_{1}=c \in[0,2]$. Then, using triangle inequality, we get

$$
\begin{aligned}
\left|a_{2} a_{4}-a_{3}^{2}\right| \leq & \frac{\left(4-c^{2}\right)}{32}+\frac{\left(4-c^{2}\right) c^{2} t^{2}}{128}+\frac{\left(4-c^{2}\right) c^{2} t}{256} \\
& +\frac{\left(4-c^{2}\right) c^{2} t^{2}}{64}+\frac{c^{4}}{1536} .
\end{aligned}
$$

Let us consider

$$
\begin{aligned}
F(c, t) & =\frac{\left(4-c^{2}\right)}{32}+\frac{\left(4-c^{2}\right) c^{2} t^{2}}{128}+\frac{\left(4-c^{2}\right) c^{2} t}{256} \\
& +\frac{\left(4-c^{2}\right)^{2} t^{2}}{64}+\frac{c^{4}}{1536} .
\end{aligned}
$$

Thus we get $\frac{\partial F}{\partial t}=\frac{\left(4-c^{2}\right) c^{2}}{256}+\frac{\left(4-c^{2}\right) c^{2} t}{64}+\frac{\left(4-c^{2}\right) t}{32} \geq 0$, which gives that $F(c, t)$ is increasing for any then $t$ in $[0,1]$. This shows that $F(c, t)$ has maximum value at $t=1$.

$$
\begin{aligned}
\operatorname{Max} F(c, t) & =F(c, 1)=\frac{\left(4-c^{2}\right) c^{2}}{256}+\frac{\left(4-c^{2}\right) c^{2}}{258} \\
& +\frac{\left(4-c^{2}\right)^{2}}{64}+\frac{\left(4-c^{2}\right)}{32}+\frac{c^{4}}{1536}
\end{aligned}
$$


Let us define $M(c):=\frac{3\left(4-c^{2}\right) c^{2}}{256}+\frac{\left(4-c^{2}\right)^{2}}{64}$ $+\frac{\left(4-c^{2}\right)}{32}+\frac{c^{4}}{1536}$, then

$M^{\prime}(c):=\frac{3\left(4-c^{2}\right) c}{128}-\frac{\left(4-c^{2}\right) c}{32}-\frac{c^{3}}{8}-\frac{c}{16}$.

If $M^{\prime}(c)$ vanishes at $c \stackrel{32}{=} 0$. A simple computation yields that $M^{\prime \prime}(c)<0$, which means that the function $M(c)$ has maximum value at $c=0$. Hence, we have

$$
\left|a_{2} a_{4}-a_{3}^{2}\right| \leq M(0)=\frac{3}{8}
$$

Theorem 10 If $f \in \mathbb{S}_{s}^{*}\left(e^{z}\right)$ then

$\left|H_{3}(1)\right| \leq \frac{90831+1121 \sqrt{118}}{166464}=0.618$.

Proof: Since $H_{3}(1)=a_{3}\left(a_{2} a_{4}-a_{3}^{2}\right)-a_{4}\left(a_{4}-\right.$ $\left.a_{2} a_{3}\right)+a_{5}\left(a_{3}-a_{2}^{2}\right)$, by applying triangle inequality, we get

$$
\begin{aligned}
& \left|H_{3}(1)\right| \leq \\
& \left|a_{3}\right|\left|a_{2} a_{4}-a_{3}^{2}\right|+\left|a_{4}\right|\left|a_{4}-a_{2} a_{3}\right|+\left|a_{5}\right|\left|a_{3}-a_{2}^{2}\right| .
\end{aligned}
$$

Now, substituting the equations (21),(24),(25) in (27) we obtain $\left|H_{3}(1)\right| \leq 0.618$.

Now, we state some results related to the class defined in Definition 2.

Theorem 11 If $f \in C_{s}\left(e^{z}\right)$ then $\left|a_{2}\right| \leq \frac{1}{4}$, $\left|a_{3}\right| \leq \frac{1}{6},\left|a_{4}\right| \leq \frac{19}{192},\left|a_{5}\right| \leq \frac{13}{120}$.

Theorem 12 If $f \in C_{s}\left(e^{z}\right)$ then $\left|a_{3}-a_{2}^{2}\right| \leq \frac{1}{6}$.

Theorem 13 If $f \in C_{s}\left(e^{z}\right)$ then

$\left|a_{2} a_{3}-a_{4}\right| \leq \frac{157446-5575 \sqrt{104}}{1168032}=0.08612$.

Theorem 14 If $f \in C_{s}\left(e^{z}\right)$ then $\left|a_{2} a_{4}-a_{3}^{2}\right| \leq \frac{25}{576}$.

Theorem 15 If $f \in C_{s}\left(e^{z}\right)$ then $\left|H_{3}(1)\right| \leq 0.0338$.

\section{References:}

[1] R.M. Ali, S. K. Lee, V. Ravichandran, S. Supramaniam, The Fekete-Szegö coefficient functional for transforms of analytic functions. Bull. Iranian Math. Soc. Vol.35, No. 2, 2009,pp. 119142.

[2] R.M. Ali, V. Ravichandran, N. Seenivasagan, Coefficient bounds for p-valent functions. Appl. Math. Comput. Vol.187, No. 1,2007, pp. 35-46.

[3] S. Altinkaya, S. Yalcin, Third Hankel determinant for Bazilevic functions, Advances in Math., 5(2016), Vol.5, No. 2, 2016, pp.91-96.
[4] K.O. Babalola, On Hankel determinant for some classes of univalent functions, Inequal. Theory Appl., Vol.6, 2007, pp. 1-7.

[5] D. Bansal, Upper bound of second Hankel determinant for a new class of analytic functions. Appl. Math. Lett. Vol. 26, No. 1, 2013, pp.103107.

[6] N.E. Cho, S. Owa, Shigeyoshi. On the FeketeSzeg $\ddot{o}$ problem for strongly $\alpha$-quasi convex functions. Tamkang J. Math. Vol.34, No. 1, 2003, pp. 21-28.

[7] N.E. Cho, S. Owa, On the Fekete-Szegö problem for strongly $\alpha$-logarithmic quasi convex functions. Southeast Asian Bull. Math. Vol. 28, No. 3, 2004, pp. 421-430.

[8] N.E. Cho, B. Kowalczyk, O.S. Kwon, A. Lecko, Y.J. Sim, Some coefficient inequalities related to the Hankel determinant for strongly starlike functions of order alpha. J. Math. Inequal. Vol.11, No. 2, 2017, pp. 429-439.

[9] R. Ehrenborg, The Hankel determinant of exponential polynomials. Amer. Math. Monthly Vol. 107 ,No. 6, 2000, pp. 557-560.

[10] M. Fekete, G. Szego, Eine Bemerkung Uber Ungerade Schlichte Funktionen. J. London Math. Soc. Vol. 8, No. 2, 1933, pp.85-89.

[11] Hai-Yan Zhang, Huo Tang, Xiao-Meng Niu, Third Order Hankel determinant for certain class of analytic functions related with exponential function, MDPI, Symmetry Vol.10, 501, 2018,doi:10.3390/sym.10100501.

[12] M. Haripriya,R.B. Sharma, On a class of bounded turning function subordinate to leaf like domain, J. Phy. Contemp Series 2018, 1000-012056, doi: 10.1088/1742-596/1000 /1/0102056.

[13] W.K. Hayman, On the second Hankel determinant of mean univalent functions. Proc. London Math. Soc. Vol. 3, No. 18, 1968, pp. 77-94.

[14] Janteng Aini, Halim Suzeini Abdul, Maslina Darus, Coefficient inequality for a function whose derivative has a positive real part. JIPAM. J. Inequal. Pure Appl. Math. Vol. 7, No. 2, 2006, Article 50, 5 pp.

[15] Janteng Aini, Halim Suzeini Abdul, Maslina Darus, Hankel determinant for starlike and convex functions. Int. J. Math. Anal. (Ruse) 1 (2007), no. 13-16, pp.619-625. 
[16] F. R. Keogh, E. P. Merkes, A coefficient inequality for certain classes of analytic functions. Proc. Amer. Math. Soc. Vol. 20, 1969, pp. 8-12.

[17] B. Kowalczyk, A. Lecko, Y.J. Sim, The sharp bound for the Hankel determinant of the third kind for convex functions. Bull. Aust. Math. Soc. Vol .97, No. 3, 2018, pp.435-445.

[18] K. Rajya Laxmi, R.Bharavi Sharma, Second Hankel determinants for some subclasses of biunivalent functions associated with pseudostarlike functions. J. Complex Anal. 2017, Art. ID 6476391, pp.1-9.

[19] A. Lecko, Y.J. Sim, B. Smiarowska, The sharp bound of the Hankel determinant of the third kind for starlike functions of order 1/2, Complex Analysis and Operator Theory,Doi.org/10.1007/s11785-018-0819-0, July 2018.

[20] S.K. Lee, V. Ravichandran, S. Supramaniam, Bounds for the second Hankel determinant of certain univalent functions. J. Inequal. Appl., 2013, 2013:281, 17 pp.

[21] Z. Lewandowski, S.S. Miller, E. Zlotkievicz, Gamma-Starlike functions. Ann. Univ. Math. J., Vol. 27, No.4, 1978, pp.671-688.

[22] R. J. Libera, E. J. Zlotkiewicz, Coefficient bounds for the inverse of a function with derivative in . Proc. Amer. Math. Soc. Vol. 87, No. 2, 1983, pp. 251-257.

[23] Liu Ming-Sheng, Xu Jun-Feng, Yang Ming, Upper bound of second Hankel determinant for certain subclasses of analytic functions. Abstr. Appl. Anal. (2014), 2014, Art.ID 603180, pp.1-10.

[24] W.C. Ma, D. Minda, A unified treatment of some special classes of univalent functions. Proceedings of the Conference on Complex Analysis (Tianjin, 1992), 157-169, Conf. Proc. Lecture Notes Anal., I, Int. Press, Cambridge,MA, 1994, pp. 157-169.

[25] N. Magesh, V. K. Balaji, Fekete-Szegö problem for a class of convex and starlike functions associated with kth root transformation using quasisubordination. Afr. Mat. Vol.29, No. 5-6, 2018, pp. $775-782$.

[26] R. Mendiratta, S. Nagpal, V. Ravichandran, On a subclass of strongly starlike functions associated with exponential function. Bull.Malays. Math. Sci. Soc. Vol. 38, No. 1, 2015, pp.365-386.
[27] Mohd Narzan Mohammed Pauzi, M. Darus, Saibah Siregar, Second and third Hankel determinant for a class defined by generalized polylogarithm functions, TJMM, Vol. 10 No. 1, 2018, pp.31-41.

[28] G. Murugusundaramurthy, S. Kavitha, T. Rosy, On the Fekete-Szego problems for Some Subclasses of analytic functions defined by convolution, Proc. Pakistan ACAD Soc., Vol.44 ,No.4,pp.249-254.

[29] J.W. Noonan, D.K. Thomas, On the second Hankel determinant of areally mean $p$-valent functions. Trans. Amer. Math. Soc. Vol. 223,1976, pp.337-346.

[30] K.I. Noor, Inayat. Hankel determinant problem for the class of functions with bounded boundary rotation. Rev. Roumaine Math. Pures Appl. Vol. 28 , No. 8, 1983, pp.731-739.

[31] H. Orhan, N. Magesh, J. Yamini, Bounds for the second Hankel determinant of certain biunivalent functions. Turkish J. Math. Vol. 40, No. 3, 2016, pp.679-687.

[32] J.K. Prajapat, D. Bansal, A. Singh, A. K Mishra, Bounds on third Hankel determinant for close-to-convex functions. Acta Univ. Sapientiae Math. Vol.7, No. 2, 2015, pp.210-219.

[33] J.K. Prajapat, D. Bansal, S. Maharana, Bounds on third Hankel determinant for certain classes of analytic functions. Stud. Univ. Babes-Bolyai Math., Vol.62, No. 2, 2017, pp.183-195.

[34] Ch. Pommerenke, Univalent functions, J. Math. Soc., Japan, Vol. 49,1975, pp.759-780.

[35] Ch. Pommerenke, On the coefficients and Hankel determinants of univalent functions. J. London Math. Soc., Vol. 41, 1966, pp. 111-122.

[36] Ch. Pommerenke, On the Hankel determinants of univalent functions. Mathematika Vol. 14,1967, pp.108-112.

[37] D. Răducanu, P. Zaprawa Second Hankel determinant for close-to-convex functions. $C$. $R$. Math. Acad. Sci. Paris Vol. 355, No. 10, 2017, pp.1063-1071.

[38] R. K. Raina, J. Sokól, On coefficient estimates for a certain class of starlike functions. Hacet. J. Math. Stat. Vol.44, No. 6, 2015, pp.1427-1433. 
[39] T. Ram Reddy, R.B. Sharma, Fekete-Szegö inequality for some subclasses of analytic functions involving complex order, Mathematical Sciences International Research Journal, Vol. 1, Issue-2, 2012.

[40] V. Ravichandran, Y. Polatoglu, M. Bolcal, A. Sen Certain subclasses of starlike and convex functions of complex order. Hacet. J. Math. Stat.Vol. 34 , 2005, pp.9-15.

[41] T. N. Shanmugam, C. Ramachandran, V. Ravichandran, Fekete-Szegö problem for subclasses of starlike functions with respect to symmetric points. Bull. Korean Math. Soc. Vol. 43, No. 3, 2006, pp. 589-598.

[42] T. Ram Reddy, R.B. Sharma, K. Saroja, A new subclass of meromorphic functions with positive coefficients. Indian J. Pure Appl. Math. Vol. 44, No. 1, 2013, pp. 29-46.

[43] H. M. Srivastava, A. K. Mishra, M.K. Das, The Fekete-Szegö problem for a subclass of closeto-convex functions. Complex Variables Theory Appl. Vol. 44 , No. 2, 2001, pp.145-163.

[44] N. Tuneski, M. Darus, Fekete-Szegö functional for non-Bazilevič functions. Acta Math. Acad. Paedagog. Nyhzi. (N.S.) Vol.18, No. 2, 2002, pp.63-65.

[45] D. Vamshee Krishna, B. Venkateswarlu, T. RamReddy, Third Hankel determinant for certain subclass of p-valent functions. Complex Var. Elliptic Equ. Vol.60, No. 9, 2015, pp. 1301-1307.

[46] D. Vamshee Krishna, B. Venkateswarlu, T. RamReddy, Third Hankel determinant for starlike and convex functions with respect to symmetric points. Ann. Univ. Mariae CurieSk todowska Sect. Vol. 70, No. 1, 2016, pp.37-45.

[47] P. Zaprawa, On Hankel determinant $H_{2}(3)$ for univalent functions. Results Math., Vol. 73, No. 3, 2018, Art. 89, pp.1-12. 